\title{
Systems Thinking: An Effective Tool for Problem Resolution and Change Management
}

\author{
Katherine L. Clinton, Tricia A. Dougherty, Tony R. Masimore \\ Lockheed Martin Maritime Systems \& Sensors (MS2)
}

\section{Introduction}

The systems development environment today demands that the processes employed become mature and are implemented in the most expeditious manner. There is a tremendous demand on the organization's human and physical assets. The organization under study realized that this was a growing issue that needed to be addressed immediately and aggressively. The issue was exacerbated by the fact that many system upgrades to the legacy system were being implemented in parallel, and the system knowledge was rapidly being depleted by attrition and the inability to retain experienced employees.

In an attempt to alleviate the problem, an automated test capability was pursued. It soon became apparent that there were underlying problems in the manual test development process that would preclude an efficient and economical implementation of a test automation capability. Manual test development processes encompass the organization's processes that govern how the following tasks are accomplished without the aid of Computer Assisted Systems Engineering (CASE) tools:

- Test requirements analysis

- Test case design

- Test procedure development

- Test execution

The manual test development processes were immature and automation would only achieve chaos that much quicker and at a greater cost:

- The processes were neither consistent nor integrated into the overall system development life cycle.

- They were not repeatable from one program to the next.

- Process excellence was dependent upon the heroic efforts of the individuals involved on a particular program.

There was a need to capture the best practices that were being employed across the organization, to apply industry standard practices and to institutionalize those practices across all programs being implemented within the organization.

\section{Background}

A set of changes were proposed to the test development process that would ensure integration with the overall system development process, consistency across the organization, and conformity with the business unit's Capability Maturity Model Integrated (CMMI) initiatives. The changes would contribute to the development of policies and procedures to support the test development process. They would also provide for periodic assessment of the process and provide mechanisms for recommendations for process modifications in areas where process improvement can be achieved.

As with any effort to change the status quo, there was a tremendous amount of resistance to be overcome. The program test lead was able to leverage the systemic thinking disciplines to identify the blockers to change, to overcome the resistance to change and to implement the proposed set of changes.

The systems thinking process of discovery and diagnosis was used by the test lead and program technical leads to explore the problem space and identify the optimal set of solutions. The application of the systems thinking principles resulted in the realization of changes that produced record-breaking program success. As a result of the change the organization's subsystems were consistently 20 percent ahead of schedule and experienced low single-digit failure rates throughout systems integration and testing.

\section{System Verification Process Problem}

The test development process life cycle should parallel that of the program development life cycle. For each stage of the program life cycle, there is an equivalent phase of the test program life cycle with assigned test activities that must be accomplished. Associated with each stage is a defined set of processes that must be identified and adhered to in order to ensure a mature and effective test program (Burnstein, Suwannasart, and Carlson 1996). This was not the case with the organization under study. 
The current process was very costly and labor intensive. The verification of functional requirements was done on a requirement-by-requirement basis. This resulted in inefficient use of test times and physical and human resources in the conduct of subsystem functional level testing.

The test development method employed by each subsystem organization varied significantly. Basic testing techniques and methods were not institutionalized. A change and a means of implementing the change were necessary.

Due to the team composition for the Integrated Product Teams (IPTs) within the organization and the project development schedule, the need for the change was evident in order to succeed. The team consisted of mostly inexperienced engineers with little to no legacy system knowledge. This composition would have far-reaching impacts on the overall quality of the product to be delivered. Therefore, a systems approach had to be employed to overcome the obstacles being faced on the program.

\section{Change Proposal Objectives}

The primary objective of the proposed change was to establish a common process across all programs that would produce a quality set of tests that are well-defined and repeatable, thus reducing overall program development costs.

The proposed changes introduced a more rigorous test development process that provided for the following:

- Increased early defect identification

- Increased test coverage

- Expanded testing beyond the "Go" path

As the organization strived to ensure that the processes defined for other system development activities were in compliance with the objectives of CMMI, it also defined test processes that were consistent with the evolving and iterative CMMI process. The proposed changes met those objectives and integrated into the system development lifecycle processes.

They ensured that the requirements, design, and development are complete and compatible. The tests that were designed using this approach were documented, configuration managed, and repeatable. As they became institutionalized, they provided the basis for commonality across various programs. The methodology provided for greater granularity in collecting metrics for continued process improvement.

\section{Systems Thinking Tools Employed for Change Implementation}

Systems thinking is a process of discovery and diagnosis. It provides the tools for an organization to inquire into the governing processes underlying the problems it faces and for representing the system (the interrelationships of the organization).

The two basic building blocks (feedback loops) of systems representations are reinforcing and balancing loops. "Reinforcing loops generate exponential growth and collapse, in which the growth or collapse continues at an ever-increasing rate" (Senge et al. 1994, 114-15). Some key characteristics and indicators of a reinforcing loop are as follows:

- Within any reinforcing processes/loops, a small change builds on itself and continues to escalate.

- The situation under study generally experiences highly amplified growth or decline.

- Phrases such as "We're going to hell in a hand basket," "We're on a downward spiral," or "We're on a roll" are commonly heard.

- The loop is incomplete by definition.

- As the situation continues to progress, eventually it runs up against at least one balancing mechanism that limits it.

Those processes that eventually limit growth are called balancing processes; they generate the forces of resistance. They are also the mechanisms in a system that fix problems, maintain stability, and achieve equilibrium when situations are "snowballing" out of control. Some of the key characteristics of a balancing process are the following:

- They are always bound to a target such as a constraint or goal that is often implicitly set by the force of the system.

- Whenever current reality does not match the balancing loop's target, a gap is naturally created.

- This resulting gap generates a kind of pressure that the system cannot ignore.

- It is at this point that the organization must recognize the gap and identify the driving goal or constraint to gain an understanding of the balancing loop.

- Phrases indicative of the balancing loop are "We're being jerked back and forth," "We're on a roller coaster," "We're running into walls," or "We can't change the system no matter how hard we try."

Delays can occur in both loop types. Delays occur when the chain of influence takes a long time to produce change. In reinforcing loops, confidence can be shaken when delays occur, because growth does not come as quickly as expected. Delays can dramatically change the behavior of the system in balancing loops and accelerate the process to desired results when properly analyzed.

Systems thinking archetypes allow an organization to show feedback (reinforcing and balancing) relationships by visually portraying the interconnected nature of its elements. The tools can be used to construct credible and consistent hypotheses about the challenges that an organization faces. Each archetype tells a story 
about the organization's problems. They energize organizational learning.

In order to apply the systems thinking archetypes to the problem being observed by this case study, the structure of the organization's system (its pattern of interrelationships among key components) was examined. This examination considered a number of factors, some of which are as follows:

- Process flows

- Prevailing attitudes and perceptions

- Product quality

- Organizational learning patterns

- Decision-making processes

The test lead had to learn to recognize the ramifications and trade-offs of the actions taken in applying the systems thinking methodology. One of the key tenets of systems thinking is that it cannot be practiced as an individual. This realization was made by the test lead early in the change process. Because successful results in a complex system depend on bringing in as many perspectives as possible, the test lead accomplished the following:

- Made sure all necessary/interrelated functions were represented.

- Gained clearance from top management to propose cross-functional solutions, despite sensitivities and politics.

- Made sure no area of the organization was off limits or protected.

- Included a variety of learning styles on the change implementation team.

- Selected team members to help facilitate change who were willing to take a stand and knew in advance that their advice would be unpopular.

- Solicited and obtained the assistance of other technical leads on the program.

The archetypes used to describe the problems (the system behavior) that the organization encountered and the solutions applied to their resolution are defined below (Senge et al. 1994, 122-23):

- Reinforcing Loop-An important variable accelerates up (or down), with exponential growth or collapse.

- Balancing Loop-There is movement toward a target (without delay), or else oscillation, hovering around a single target (with delay).

- "Fixes That Backfire"-A problem symptom alternately improves (the problem variable goes down) and deteriorates (the problem goes up, worse than before).

n "Limits to Growth"-There is growth (sometimes dramatic growth), leveling off or falling into decline.

n "Shifting the Burden"-Three patterns exist side by side. The reliance on the short-term fix grows stronger, while efforts to fundamentally correct the real problem grow weaker, and the problem symptom alternately improves and deteriorates.
- "Tragedy of the Commons" - Total activity grows, but the gains from individual activities drop.

- "Accidental Adversaries"-Each side's performance either declines or stays level and low, while enmity or competitiveness increases over time.

Most of the archetypes are related strategically to each other. Use of the archetypes helps avoid finding the easiest and fastest solutions. The easiest solutions will lead back in; the intangible elements of the system are where the leverage for effective change increases. The stories of the organization under study represented by the archetypes defined above are described in the section entitled "Systems Thinking Case Study Overview."

\section{Methodology}

The program test lead was able to leverage the systemic thinking discipline to identify the blockers to change and to overcome the resistance to change. "Organizations learn only through individuals who learn. Individual learning does not guarantee organizational learning. But without it no organizational learning occurs" (Lawson 2003). These statements are descriptive of the organization under change: Systems dynamics pointed out the vulnerabilities, limited understandings and fallibilities of the past and the assurance that today's thinking will be the source of tomorrow's problems. True to the nature of systems dynamics, the discovery process led to confrontation as awareness was raised.

Implementation of changes to the current test program required learning, first and foremost, on the part of the test lead. The lead realized the importance of the personal mastery discipline, which enabled the articulation of the vision for change to the directorate management and the implementation team.

In the face of opposition, the test lead began to articulate the vision through commitment and an energetic pursuit. It required that the test lead step back and take the time to understand how the organization had arrived at the current testing methodology, obtain a greater appreciation for the frustration that was being experienced and develop patience in trying to obtain acceptance of the new approach. Prior to that occurring, the organization's test lead experienced emotional tension to the point of thinking that it would be impossible to implement the vision within the organization. However, as understanding increased, creative tension was experienced which resulted in becoming more energized by the vision and regaining enthusiasm.

Through the application of protocols for exploring mental models, the team was able to expose the models that were hindering acceptance and progress. The protocols were used in conversations with SMEs, program technical leads 
and team members to determine what their opposition was to the approach and to identify possible modifications to the approach that would allow for a more disciplined methodology without becoming overburdened in the current program. It was decided that the inexperienced engineers would use the more rigorous test-development process to facilitate knowledge acquisition and the current SMEs would support the unstructured testing that gives rise to a different category of errors. Employing the reflection and inquiry skills to arrive at this decision helped train the team lead and members (Lawson 2003).

It was discovered that shared vision is only achievable through accomplishing proficiency in the personal mastery and mental models disciplines. Although the team initially received top-level management buy-in, energy rose from the bottom up. This was accomplished by bridging the gap between shared vision and current reality. Actions were taken to reduce the influence of a couple of disgruntled employees on the more impressionable team members. By giving those employees who were willing to learn more responsibility and asking for their inputs on the process, their excitement began to increase and they started to take ownership of the process.

Once those measures were set in place, team learning began to take place. Action was taken to align team members to facilitate the learning process. Listening to the team members resulted in an approach that was achievable within current program schedule and budget constraints.

Applying the reflection and inquiry skills in managing the relationship between the IPT lead and the team members also helped bridge some of the chasms that had been created. Learning to manage upward and learning to anticipate the expectations of the IPT lead and arriving at an agreed upon level of support helped decrease the test lead's personal emotional tension. The skills were consistently applied to identify means of reducing the emotional tension resulting from the relationship between the IPT lead and the team members.

\section{Systems Thinking Case Study Overview}

The change process was consistent with several archetypes as various elements of the system were examined. The two primary elements that were observed are the human system and the physical system.

\section{System Element Models}

Initially the human system story could be told by using the reinforcing loop archetype. There was a rapid decay in attitudes due to the relationship with the IPT lead and the impact that disgruntled employees were having on the entire team. The perceived impression of the disgruntled camp was that the new processes would introduce too much work to be accomplished by the engineers. There was also the impression that any process improvement initiative introduces unnecessary overhead, thus hindering progress. The reinforcing loop is depicted in Figure 1. Through the application of systemic thinking disciplines, the team was made to realize the benefits of a more rigorous and structured approach to test design and development. Some of those benefits are as follows:

- Development of skill sets that are readily transferable to other programs

Engineers that are more marketable within the systems engineering discipline

- A structured manner to become SMEs in their functional area

- Reduction in the amount of lost test time due to improper test environment setups

- Verification of requirements across multiple elements during a single test event

Figure 1. Reinforcing Loop of Human System

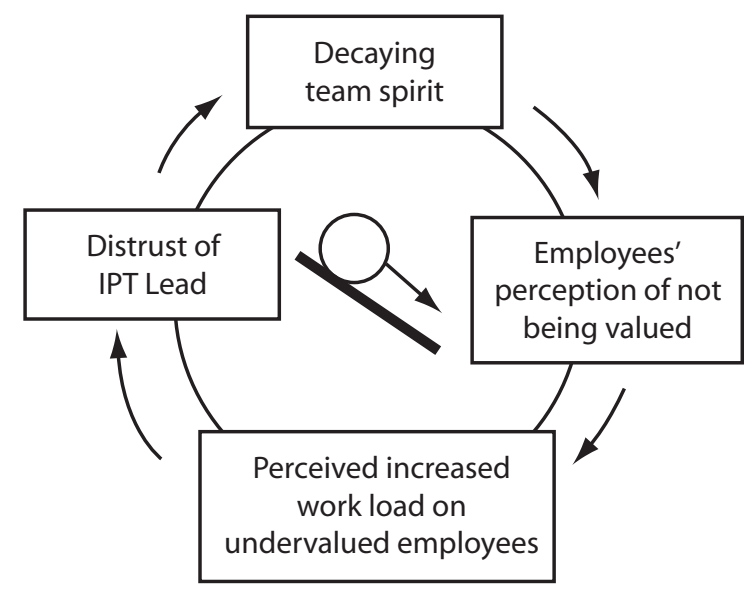

The realization brought on by the integration of systemic disciplines allowed the team to enter a balancing loop as depicted in Figure 2. In the balancing loop, the team was able to gain momentum and enter into another reinforcing loop, thereby, achieving exponential acceptance/growth within the team.

During the observation stages, it was realized that productivity utilizing the existing processes was modeled by the "Tragedy of the Commons" archetype. A flurry of activity occurred on the program, but the productivity from the individual efforts declined. Some of this resulted from feelings of futility on the part of the engineers, which is indicative of immature processes.

Observations of the physical system indicated that the prevailing archetype was the shifting of the burden loop. 
Figure 2. Balancing Loop of Human System

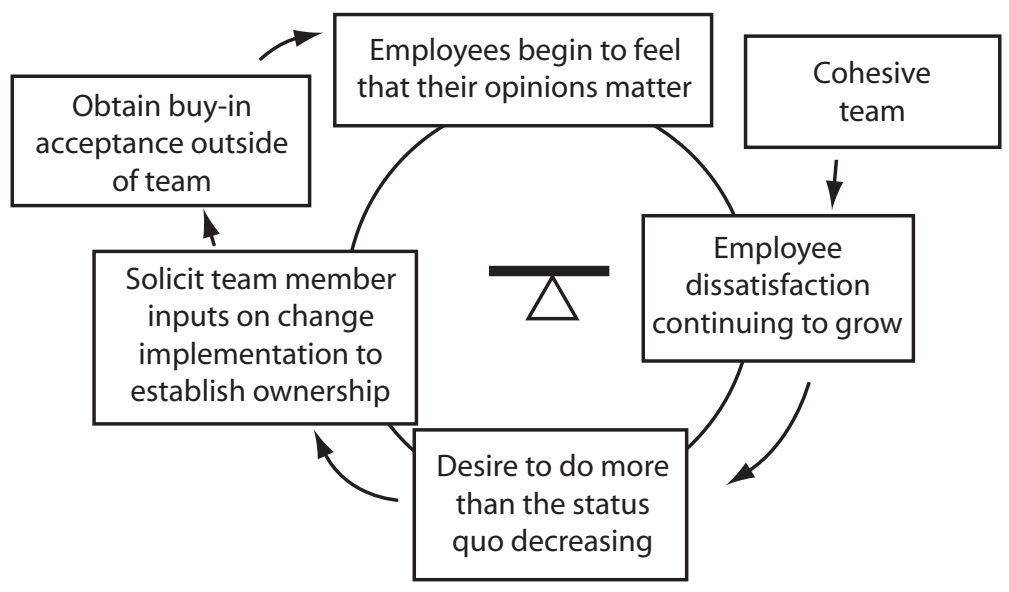

There was a tremendous need to develop young talent and establish a pipeline of SMEs in order to preserve legacy knowledge. The need was apparent, but due to program demands and limited resources, efforts were not committed to resolving the problem. Most programs limped along and struggled to meet their objectives with inexperienced engineers until the program's priority and visibility was raised to a level high enough to demand attention. The obvious solution was sought. It was only at this point that established SMEs were brought on to save the day. This behavior pattern diverted attention from establishing a mentoring program and reinforced a great dependency on "Heroes."

The physical knowledge base was in a reinforcing loop on the path to collapsing. There were no mechanisms in place to establish and maintain a repository of legacy system knowledge. Neither was there a formal and consistent way to capture lessons learned or engineering best practices that had been experienced on previous programs. Although there had been numerous amounts, there was no way to ensure that those practices were institutionalized. The proposed changes were an initial attempt to begin establishing a process for institutionalization.

The current processes and the changes implemented via CMMI and the proposal documented in this paper created a "Limits to Growth" scenario. There had been growth, sometimes dramatic growth. However, that growth had begun leveling off and in some instances falling into decline. The current processes had taken the organization to a level of growth that required change in order to proceed and realize exponential growth in the markets into which it had been expanding. The CMMI initiative had been the organization's way of preparing for that growth. However, the application of CMMI practices met with resistance within the engineering community.

In order to overcome the opposition to accepting the transition to CMMI, a mental analogy was used to explain the need for processes within a business environment.
The need for processes was compared to the need for a value system within a family unit or the individual's life. Without a value system, it is impossible to make right and wrong decisions. The value system becomes the compass for one's life and ensures that a desired course is followed to achieve the individual's goals. Processes within a business serve as that organization's "value system" in the conduct of business to achieve its goals and objectives.

\section{Change Management Model}

The change management model for implementation of changes to the test development process is identified in Figure 3 (Lawson 2003). The organization's top-level management provided the change management for the test process improvement initiative. Through observation and understanding the behavior of the current system, data and information were gathered about the current operational experiences of the system-of-interest. This

Figure 3. Change Management Model

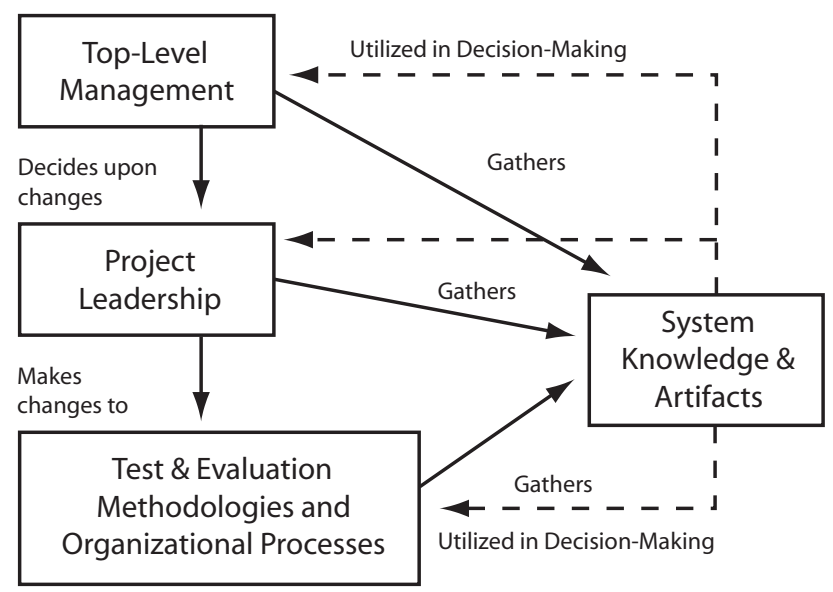


data and information were used to propose a set of changes by which management could make a decision and plan for changes to the current development method. The adaptation of the methodology required structural as well as operational changes.

Once the decision was made by management to proceed with the changes, the responsibility for executing the change was then levied upon the organization's program leadership team. Arriving at the tactical decision to make changes to the current ways of operation followed the steps of the Observation, Orientation, Decision, and Action (OODA) Loop (Lawson 2003).

As the organization leadership team began to execute the decision to change, they entered into a Plan, Do, Check, and Act (PDCA) Loop utilizing the gathered knowledge in order to strategically plan the details of the change. Based on guidelines from management, measures were established that would be used in verifying the accomplishment of the objectives of the change. Members of the program team continuously assessed the results of prescribed activities and made recommendations for changes as the group continued to iterate through the steps of the integrated loops, as depicted in Figure 4 (Lawson 2003).

As success was realized through implementation of the changes, the repository of system knowledge was established. Thus, a method of capturing legacy knowledge was set in place, allowing the organization to reduce the learning curve of new engineers. The accomplishment of this goal resulted in reduced system development costs and increased quality of the resultant products.

\section{Analysis}

The results realized by the organization under observation were consistent with those indicated by the systems thinking models. As strategies were considered, the systems thinking methodologies were used to reevaluate them. Some results of the application by the change implementation team were the following:

- A series of reinforcing and balancing loops were entered until the team's technical leads were able to obtain organizational consensus on the optimal solution set.

- Problem resolution strategies matured/evolved as the leads became more comfortable with the use of the tools.

- Began to see things within the organizational setting in four levels of simultaneous operations: (1) events, (2) patterns of behavior, (3) systems, (4) mental models.

There were a few events that occurred consistently and were key contributors to the problems being experienced. The events were analyzed during the strategy sessions and continued to surface as elements on the physical system's reinforcing loop. The persistent elements were the following:

- High first-time failure rates during testing

- Inexperienced/untrained engineers

- Longer period of time before systems are ready for inservice use due to product quality

- Schedule slips

Figure 4. Integrated OODA and PDCA Change Implementation Loop

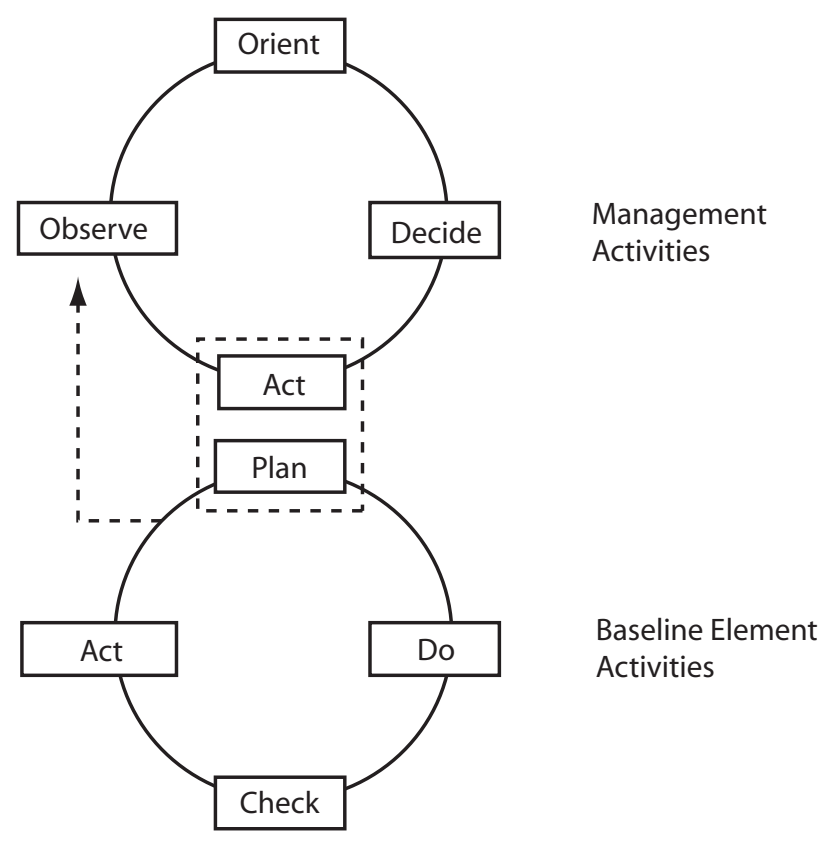


Certain patterns of behavior were recognized as occurring simultaneously as the persistent event elements spiraled out of control:

- Fewer experienced or mentored personnel were allocated upfront to a program.

- Lack of knowledge transfer upfront on programs helped contribute to the high first-time failure rates.

- There was slow progress in I\&T.

- The organization experienced a tremendous amount of overtime with low productivity results.

- There was a decrease in team morale.

Analysis of the organization's systems uncovered a number of causal relationships resulting from the simultaneous operation of the events, patterns of behavior, and mental models that existed within the system. The relationships between each of those factors were modeled by the archetypes. The systems thinking tools were used to help shift the mental models that existed and take advantage of creative tension to identify solution sets.

As organizational learning was energized through the application of systems thinking methodologies among the team under study, team learning increased and produced results that were consistent with experienced engineers (and in many cases exceeded those), as depicted in Table 1.

The productivity results of the team under study are represented by the Proj B statistics in the table. The results represented by Proj A are averages indicative of results experienced by previous project teams where the more experienced engineers were used or "Heroes" were brought on late in the program schedule to "save the day."

The team under study consisted of fewer engineers with less engineering experience and, on average, less legacy system knowledge. Applying methods employed by the technical leads during reflection and inquiry helped to accelerate the knowledge acquisition process. The junior engineers acquired knowledge quickly; learning improved exponentially compared to knowledge acquisition on other programs. This was more noticeable in the case of a cooperative education student who became a SME in four months for a functional area where one did not exist due to attrition. The change implementation was in progress when the student joined the team. It was during the time when organizational learning had been energized.

Less experienced engineers that had worked on other programs attested to the accelerated knowledge acquisition as a result of the new test development process. An independent audit of the program by a senior engineer with more than fifteen years of system experience found that the proposed changes were effective and definitely contributed to delivery of a higher quality product.

Through the application of the solution set, the team members were more prepared for test execution; thus, the test execution was more efficient, less error-pronged and more productive. The productivity levels were five times higher than that of teams consisting of more experienced engineers, as indicated by the Productivity column of Table 1 .

The organization's subsystems that implemented the proposed changes contained in this study were consistently 20 percent ahead of schedule in test execution and experienced low single-digit first-time failure rates as compared to double-digit failure rates on previous programs. The results of test execution are documented in Figure 5. A comparison of first-time failure rates as experienced by the team under study to the average rates experienced by other programs is contained in Figure 6. Another deduction that can be drawn, in addition to product quality, is that the less experienced engineers that have applied the proposed changes are now more knowledgeable of the system under test and are experiencing fewer failures during test execution.

Even when the unavailability of system assets and resources delayed the start of test execution, dramatic results were realized through the application of the changes. Those test execution results are depicted in Figure 7.

The organization agreed upon a set of recommended solutions and proceeded to implement the changes. It instituted another test methodology on top of the

Table 1. Team Productivity Results

\begin{tabular}{lccccc}
\hline Project & Number of Engineers & $\begin{array}{c}\text { Systems } \\
\text { Engineering } \\
\text { Experience }\end{array}$ & $\begin{array}{c}\text { Legacy } \\
\text { System } \\
\text { Experience }\end{array}$ & Productivity & Gains \\
\hline Proj A SS1 & 10 & 2.5 & 2 & 4.1 & \\
Proj B SS1 & 7 & 2.3 & 2.14 & 20.6 & $503.70 \%$ \\
Proj A SS2 & 20 & 2.6 & 1.7 & 3.8 & $571.60 \%$ \\
Proj B SS2 & 9 & 2.1 & 1.6 & 21.7 & \\
\hline
\end{tabular}


Figure 5. Application Results

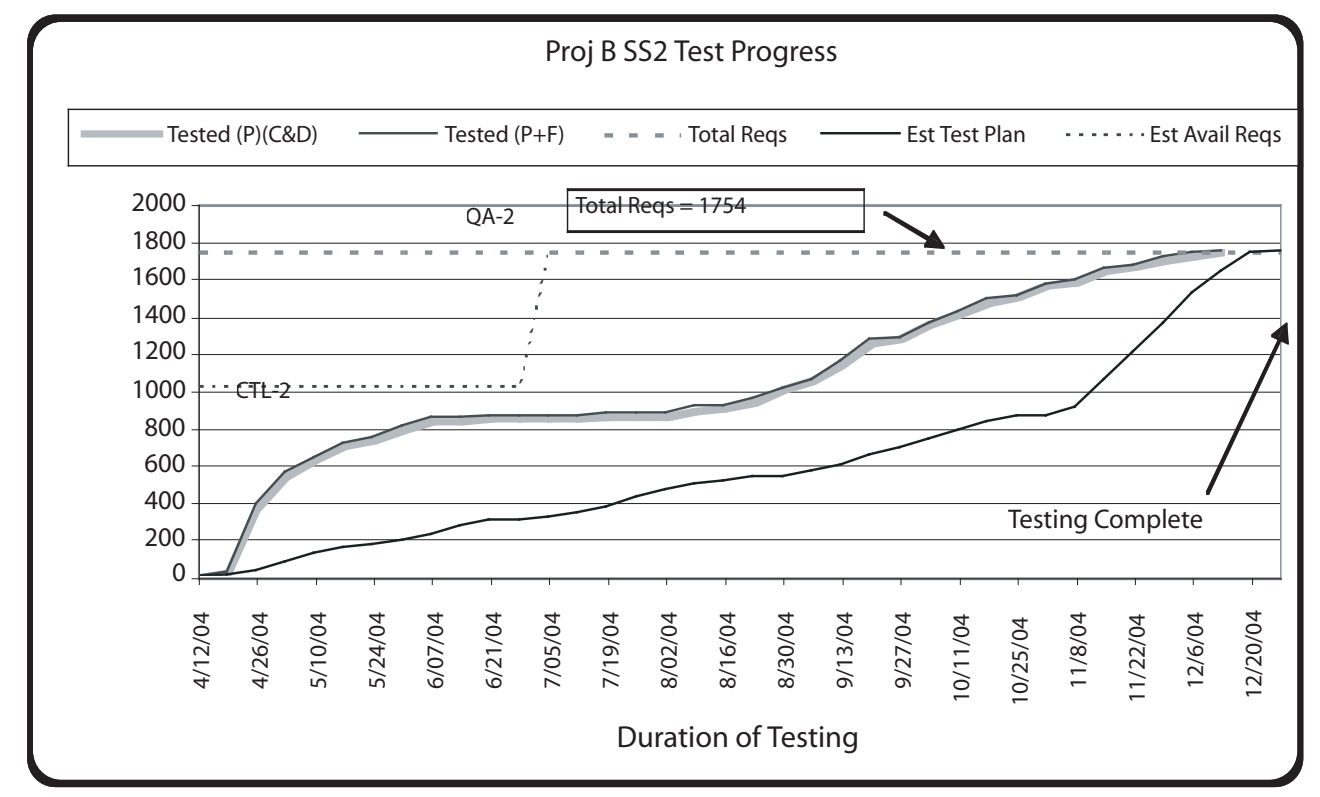

Figure 6. Historical Failure Rates

Historical Failure Rates

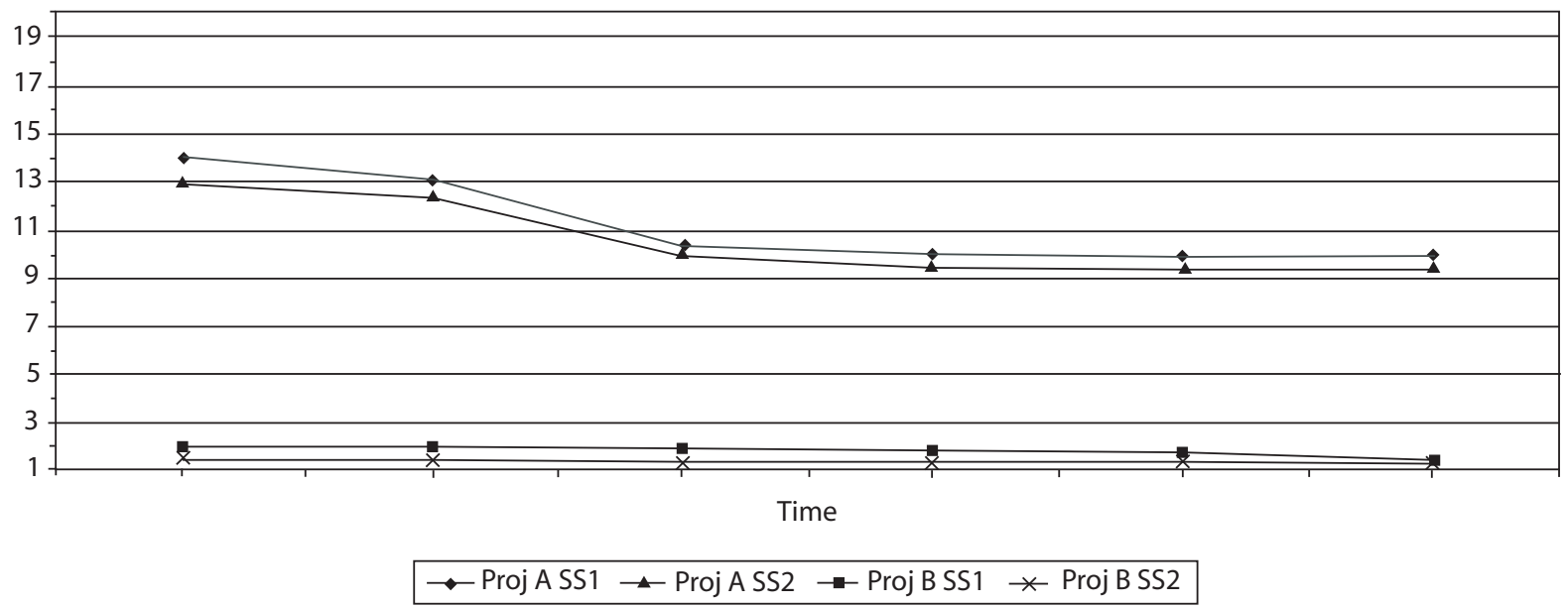

current processes to provide more control, to monitor, and provide feedback into the process. Testing became a more measured and quantified process. Reviews at all stages of the development life cycle became recognized as testing and quality control activities. System products were tested for quality attributes such as reliability, usability, and maintainability.

Industry-accepted test design methodologies were employed to improve the process to produce welldefined and repeatable tests (Hanna 1996). The test cases developed via those methodologies provided the basis for establishing test goals and the test artifacts repository/ knowledge database. This ultimately became a method of preserving legacy knowledge, which subsequently relieved the drain on system knowledge due to attrition and employee retention problems.

\section{Solutions, Implications, Recommendations}

Systems thinking is an effective tool for change management. It readily reveals hindrances to change and reveals solutions that produce the greatest impact. It is a mechanism for gaining understanding of the various aspects of the system-of-interest, reevaluating strategies, 
Figure 7. Results with System Impacts

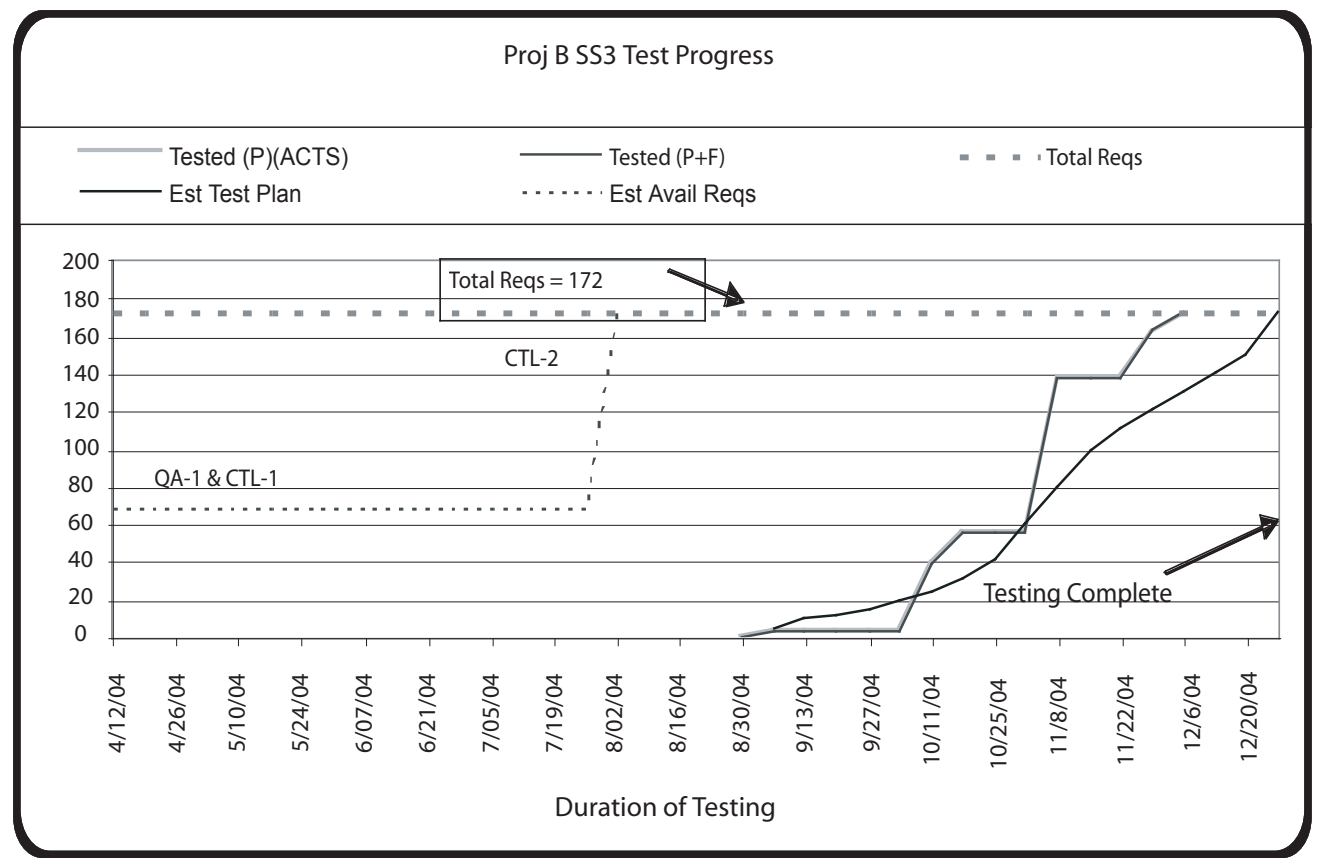

and developing tactical plans for change implementation. Its application provides clarity to development efforts and aids in the realization of results. The principles were used in assessing the current test and verification system being used by the systems engineering organization under study and in proposing changes to the test development methodologies of the organization.

The test lead obtained successful buy-in from management through a few strategic actions:

- Selected an issue that was important to the test lead and the organization.

- Chose a persistent problem for which management desired a solution.

- Bounded the scope of the problem to the test design and development practices.

- Chose a problem whose history was known and which could be described (systems archetypes).

- Chose a problem that has been tackled before, with little or no success.

- Made sure the description of the problem was as accurate as possible.

- In presenting the problem statement to management, did not leap to conclusions by including a suggestion of the solution in the problem statement.

- Remained non-judgmental throughout the process.

Systems thinking tenets dictate that it cannot be practiced by an individual. Successful results in a complex system are dependent on bringing in as many perspectives as possible. The lessons learned by the test lead in this case study and that applies to any change implementation process are as follows:

- Make sure all necessary/interrelated functions are represented in the problem identification/resolution process.

- Gain clearance/support from top management when proposing cross-functional solutions, regardless of sensitivities and politics.

- During the problem identification/resolution process, do not cut off or protect any area of the.

- In order to achieve the optimal or most feasible solution, include a variety of learning styles on the change implementation team.

- Select team members who are willing to take a stand and know in advance that their advice will be unpopular.

- Those within an organization making recommendations for change must take part in the change in order to gain a systems understanding.

- Unless there is active participation in the change implementation, the system will continually sabotage well-intentioned efforts.

The application of the principles resulted in recordbreaking program success. The organization under study has begun institutionalizing the methodologies and changes proposed within this case study on subsequent programs. As realized by this organization, systems thinking practices provide clarity to the problem space and help to identify the optimal set of solutions to the problem. 


\section{References}

Burstein, Ilene, Taratip Suwannasart, and C.R. Carlson. 1996. "Developing a testing maturity model, part II." Crosstalk The Journal of Defense Software Engineering (September).

Hanna, Magdy. 1996. Software test design and techniques. Software Dimensions.

Lawson, H.W. 2003. "Essentials of systems and their life cycles." Unpublished draft version.

Senge, P.M., A. Kleiner, C. Roberts, R.B. Ross, and B.J. Smith. 1994. The fifth discipline fieldbook-Strategies and tools for building a learning organization, New York: Doubleday.

\section{Biography}

Katherine L. Clinton is a lead member engineering staff at Lockheed Martin MS2 in Moorestown, NJ. She is currently serving as the integrated product team lead for the Aegis Ballistic Missile Defense 4.0 Mission Planner. She received her BA degree in mathematics and computer science from Albany State University, Albany, GA, and a M.S. in computer science from Florida Institute of Technology, Melbourne, FL. She is currently pursuing a Ph.D. in systems engineering from Stevens Institute of Technology in Hoboken, NJ.

Tricia Dougherty is a systems engineer for Lockheed Martin Corporation. She has worked on network modeling, design and testing for the Aegis Combat System. Tricia is currently working on modifications to the Ballistic Missile Defense system. She has a B.S. in computer science from Rutgers University and an M.S. in computer science from New Jersey Institute of Technology.

Tony Masimore is a systems engineer for Lockheed Martin Corporation. He has worked on process improvement projects and software test automation for designing and testing the Aegis Combat System. Currently, he is leading system development and test efforts for the Aegis Display Systems. He has B.A./B.S. degrees in engineering from Elizabethtown College and Pennsylvania State University and a M.S.E. in systems engineering from the University of Pennsylvania. 